\title{
Ökonomische Methoden im Recht
}

\author{
Eine Einführung für Juristen \\ Mit Beiträgen v. Markus Englerth, Sebastian J. Goerg, Stefan Magen, Alexander \\ Morell u. Klaus Ulrich Schmolke
}

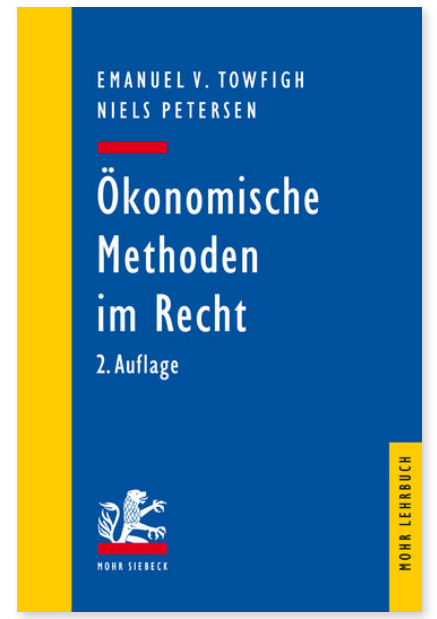

2., überarbeitete und aktualisierte Auflage; 2017. XXIV, 291 Seiten. MLB

ISBN 978-3-16-155193-2

DOI 10.1628/978-3-16-155193-2

eBook PDF

ISBN 978-3-16-155192-5

Broschur 29,00€
Ökonomische Argumente werden auch für Juristen immer wichtiger. Dieses Lehrbuch soll Juristen mit ökonomischen Methoden vertraut machen, um ihnen ein besseres Verständnis dieser Argumente zu geben. Es richtet sich dabei sowohl an Studierende als auch an Wissenschaftler und Praktiker.

Für die Neuauflage wurden alle Kapitel überarbeitet und ergänzt, zwei Kapitel sind von neuen Autoren vollständig neu verfasst worden. Außerdem wurden Randziffern eingefügt, die die Arbeit mit dem Werk erleichtern sollen.

»Im Ergebnis bietet das Buch den perfekten Einstieg in die Rechtsökonomie und darüber hinaus in die Eckpfeiler der Wirtschaftswissenschaften als solche. Das Lehrbuch bietet jedoch weit mehr. Durch die ausführlichen Literaturhinweise zu Beginn eines jeden Kapitels sowie den übersichtlichen Glossar am Ende des Werkes, wird es zu einem ständigen Begleiter. Mithin eine brillante Darstellung, die in jedes Buchregal eines Rechtsstudierenden gehört.« David Eckner www.studjur-online.de (01/2011)

"Sowohl dem auf schnellem Zugriff angewiesenen Leser als auch dem nach Vertiefung suchenden Rechtsökonomen bietet das Werk eine dem Blickwinkel des Juristen entgegenkommende Darstellung. [...] Studenten, die nach Blicköffnung und Hilfestellungen für interdisziplinär abgesicherte und nachhaltig belastbare Argumentation suchen, sei es mit Nachdruck empfohlen."

Patrick C. Leyens Juristische Schulung - 2011, Heft 1, XVII

Emanuel V. Towfigh Geboren 1978; Studium der Rechtswissenschaften in Münster und Nanjing; 2005 Promotion zum Dr. iur.; nach dem Referendardienst Post-Doc am Max-Planck-Institut zur Erforschung von Gemeinschaftsgütern in Bonn, an der New York University und an der University of Virginia; 2014 Habilitation; seit 2016 Inhaber des Lehrstuhls für Öffentliches Recht, Empirische Rechtsforschung und Rechtsökonomik an der Law School und Professor für Rechtsökonomik an der Business School, EBS Universität Wiesbaden.

Niels Petersen Geboren 1978; Studium der Rechts- und Sozialwissenschaften in Münster, Genf und New York; 2008 Promotion zum Dr. iur.; Wissenschaftlicher Referent am Max-Planck-Institut zur Erforschung von Gemeinschaftsgütern; Inhaber des Lehrstuhls für Öffentliches Recht einschließlich Völker- und Europarecht der Universität Münster.

Jetzt bestellen:

https://mohrsiebeck.com/buch/oekonomische-methoden-im-recht-9783161551932?no_cache=1 order@mohrsiebeck.com

Telefon: +49 (0)7071-923-17

Telefax: +49(0)7071-51104 